\title{
Error Estimates for the Cardinal Spline Interpolation
}

\author{
Gennadi Vainikko
}

\begin{abstract}
For the Sobolev class $W_{\text {per }}^{m, \infty}(\mathbb{R})$ of 1-periodic functions, an unimprovable error estimate for the spline interpolants of order $m$ on the uniform grid is known. In the present paper, this error estimate is extended to the Sobolev class $V^{m, \infty}(\mathbb{R})$ of (nonperiodic) functions on $\mathbb{R}$ having bounded $m$ th derivative. Some further error estimates are established including the error estimates for derivatives of the spline interpolant.
\end{abstract}

Keywords. Splines, interpolation, error estimates, best constants

Mathematics Subject Classification (2000). 41A15, 41A05, 41A44

\section{Introduction}

For a function $f \in C(\mathbb{R})$ which is bounded or at most of a polynomial growth as $|x| \rightarrow \infty$, let $Q_{h, m} f$ be the cardinal interpolant [4-6] of $f$ by splines of order $m$ (or of degree $m-1$ ) with spline knots $i h$ and interpolation knots $\left(i+\frac{m}{2}\right) h$, $i \in \mathbb{Z}, h>0$. A result of [2] tells that, for an 1-periodic function $f \in W_{\text {per }}^{m, \infty}(\mathbb{R})$ and $h=\frac{1}{n}$ with an even $n \in \mathbb{N}$, it holds

$$
\left\|f-Q_{h, m} f\right\|_{\infty} \leq \Phi_{m+1} \pi^{-m} h^{m}\left\|f^{(m)}\right\|_{\infty}
$$

where $\Phi_{m+1}$ is the Favard constant (see (24) below). This estimate is the best possible for the Sobolev class $W_{\text {per }}^{m, \infty}(\mathbb{R})$. The main result of the present paper states that estimate (1) with any real $h>0$ holds true also for (non-periodic) functions $f \in V^{m, \infty}(\mathbb{R})$, i.e., for any $f$ with bounded $m$ th derivative. Moreover, among all approximations using the same information as $Q_{h, m} f$, the spline interpolation yields the best result for the classes $W^{m, \infty}(\mathbb{R})$ and $V^{m, \infty}(\mathbb{R})$.

The paper is organised as follows. Section 2 contains preliminaries: we recall some properties of the cardinal father B-spline and Euler perfect splines, and we recall a fundamental result concerning the existence, uniqueness and

G. Vainikko: Institute of Applied Mathematics, University of Tartu, J. Liivi 2, 50409 Tartu, Estonia; gennadi.vainikko@ut.ee 
construction of bounded/polynomially growing interpolation splines. Section 3 is central in this paper. We first reprove estimate (1) in the periodic case in a formulation slightly different from that in [2]; our proof is more simple and more complete than the original proof but we still use the ideas of [2]. After that we extend estimate (1) to the general (non-periodic) case. In Section 4 we discuss the optimality of estimate $(1)$ on the classes $V^{m, \infty}(\mathbb{R})$ and $W^{m, \infty}(\mathbb{R})$. Sections 5-7 are devoted to the error estimates of the spline interpolation in the case of modestly smooth functions $f$ and to error estimates for the derivatives of the spline interpolant.

Without proofs, the results of the paper have been announced in conference work [9]. About error estimates for quasi-interpolants based on (1) see [3].

We use the standard notations $\mathbb{N}=\{1,2, \ldots\}, \mathbb{Z}=\{\ldots,-2,-1,0,1,2, \ldots\}$, $\mathbb{R}=(-\infty, \infty) ; \mathbb{C}$ is the set of complex numbers, $\mathcal{P}_{m}$ is the set of polynomials of degree $\leq m$. As usual, $C(\mathbb{R})$ is the space of continuous functions on $\mathbb{R}$, and $C^{m}(\mathbb{R})$ is the space of functions on $\mathbb{R}$ that have continuous derivatives up to the order $m$. By $B C(\mathbb{R})$ we mean the Banach space of bounded continuous functions $f$ on $\mathbb{R}$ equipped with the norm $\|f\|_{\infty}=\sup _{x \in \mathbb{R}}|f(x)|$, and $B U C(\mathbb{R})$ is the (closed) subspace of $B C(\mathbb{R})$ consisting of bounded uniformly continuous functions on $\mathbb{R}$. The Sobolev space $W^{m, \infty}(\mathbb{R}), m \in \mathbb{N}$, consists of $f$ such that $f$ itself and its derivatives up to the order $m$ are measurable and bounded in $\mathbb{R}$ (actually then $f, f^{\prime}, \ldots, f^{(m-1)}$ are continuous in $\mathbb{R}$; the derivatives are understood in the sense of distributions). Finally, the Sobolev space $V^{m, \infty}(\mathbb{R})$ consists of functions $f$ such that $f^{(m)}$ is measurable and bounded in $\mathbb{R}$; then $f, f^{\prime}, \ldots, f^{(m-1)}$ are continuous but not necessarily bounded in $\mathbb{R}$. With the help of the Taylor formula

$$
f(x)=\sum_{l=0}^{m-1} \frac{f^{(l)}(0)}{l !} x^{l}+\frac{1}{(m-1) !} \int_{0}^{x}(x-t)^{m-1} f^{(m)}(t) d t, \quad x \in \mathbb{R},
$$

we observe that for $f \in V^{m, \infty}(\mathbb{R}),|x| \rightarrow \infty$, it holds

$$
\left|f^{(k)}(x)\right| \leq \frac{1}{(m-k) !}\left\|f^{(m)}\right\|_{\infty}|x|^{m-k}+O\left(|x|^{m-k-1}\right), \quad k=0, \ldots, m-1 .
$$

Clearly, $W^{m, \infty}(\mathbb{R})+\mathcal{P}_{m} \subset V^{m, \infty}(\mathbb{R})$; this inclusion is strict. We do not need norms in $W^{m, \infty}(\mathbb{R})$ and $V^{m, \infty}(\mathbb{R})$.

\section{Preliminaries}

2.1. The father B-spline. The father B-spline $B_{m}$ of order $m$ in the terminology of $[1,7]$, or of degree $m-1$ in the terminology of $[2,8,10]$ can be defined 
by the formula

$$
B_{m}(x)=\frac{1}{(m-1) !} \sum_{i=0}^{m}(-1)^{i}\left(\begin{array}{c}
m \\
i
\end{array}\right)(x-i)_{+}^{m-1}, \quad x \in \mathbb{R}, m \in \mathbb{N},
$$

where, as usual, $0 !=1,0^{0}:=\lim _{x \downarrow 0} x^{x}=1,\left(\begin{array}{c}m \\ i\end{array}\right)=\frac{m !}{i !(m-i) !}$,

$$
(x-i)_{+}^{m-1}:= \begin{cases}(x-i)^{m-1}, & x-i \geq 0 \\ 0, & x-i<0 .\end{cases}
$$

Let us list some propeties of $B_{m}$ for $m \geq 2$ :

$$
\left.B_{m}\right|_{[i, i+1]} \in \mathcal{P}_{m-1}, i \in \mathbb{Z}, \quad B_{m} \in C^{(m-2)}(\mathbb{R}),
$$

i.e., $B_{m}$ is a spline of defect 1 , degree $m-1$ on the "cardinal" knot set $\mathbb{Z}$,

$$
\begin{aligned}
B_{m}^{\prime}(x) & =B_{m-1}(x)-B_{m-1}(x-1), & & x \in \mathbb{R}(x \neq 0,1,2 \text { in case } m=2) \\
B_{m}^{(m-1)}(x) & =(-1)^{i}\left(\begin{array}{c}
m-1 \\
i
\end{array}\right) & & \text { for } i<x<i+1, i=0, \ldots, m-1
\end{aligned}
$$

and

$$
\begin{gathered}
\operatorname{supp} B_{m}=[0, m], \quad B_{m}(x)>0 \text { for } 0<x<m, \quad \int_{\mathbb{R}} B_{m}(x) d x=1 \\
B_{m}\left(\frac{m}{2}-x\right)=B_{m}\left(\frac{m}{2}+x\right), x \in \mathbb{R}, \quad B_{m}\left(\frac{m}{2}\right)=\max _{x \in \mathbb{R}} B_{m}(x) \\
\sum_{j \in \mathbb{Z}} B_{m}(x-j)=1, x \in \mathbb{R} .
\end{gathered}
$$

2.2. Wiener interpolant. In this section we recall some fundamental results [4-6], [8] concerning the existence, uniqueness and construction of the cardinal interpolation splines. We accent the relation between the interpolation and the Wiener theorem about Fourier/Laurent series.

Introduce in $\mathbb{R}$ the uniform grid $h \mathbb{Z}=\{i h: i \in \mathbb{Z}\}$ of the step size $h>0$. Denote by $S_{h, m}, m \in \mathbb{N}$, the space of splines of order $m$ (of degree $m-1$ ) and defect 1 with the knot set $h \mathbb{Z}$. The dilated-shifted B-splines $B_{m}\left(h^{-1} x-j\right)$, $j \in \mathbb{Z}$, cf. (4), (5), belong to $S_{h, m}$, and the same is true for $\sum_{j \in \mathbb{Z}} d_{j} B_{m}\left(h^{-1} x-j\right)$ with arbitrary coefficients $d_{j}$; this series is locally finite: due to (8),

$$
\sum_{j \in \mathbb{Z}} d_{j} B_{m}\left(h^{-1} x-j\right)=\sum_{j=i-m+1}^{i} d_{j} B_{m}\left(h^{-1} x-j\right) \quad \text { for } x \in[i h,(i+1) h), i \in \mathbb{Z} .
$$

Given a function $f \in C(\mathbb{R})$, let us look for the interpolant

$$
f_{h, m}(x)=\sum_{j \in \mathbb{Z}} d_{j} B_{m}\left(h^{-1} x-j\right), \quad x \in \mathbb{R},
$$


determining the coefficients $d_{j}$ by the interpolation conditions

$$
f_{h}\left(\left(k+\frac{m}{2}\right) h\right)=f\left(\left(k+\frac{m}{2}\right) h\right), \quad k \in \mathbb{Z} .
$$

This leads to the bi-infinite system of linear equations

$$
\sum_{j \in \mathbb{Z}} b_{k-j, m} d_{j}=f_{k}, \quad k \in \mathbb{Z}
$$

where

$$
b_{k, m}=B_{m}\left(k+\frac{m}{2}\right), \quad f_{k}=f_{k, h, m}=f\left(\left(k+\frac{m}{2}\right) h\right), \quad k \in \mathbb{Z} .
$$

Denote also

$$
\mu:=\operatorname{int}\left(\frac{m-1}{2}\right)= \begin{cases}\frac{m-2}{2}, & m \text { even } \\ \frac{m-1}{2}, & m \text { odd. }\end{cases}
$$

It follows by (8)-(10) that

$$
b_{k, m}=\left\{\begin{array}{ll}
b_{-k, m}>0 & \text { for }|k| \leq \mu \\
0 & \text { for }|k|>\mu,
\end{array} \quad \sum_{|k| \leq \mu} b_{k, m}=1 .\right.
$$

Thus (14) is a system with the Toeplitz band matrix $\mathfrak{B}_{m}=\left(b_{k-j, m}\right)_{k, j \in \mathbb{Z}}$ of the band width $2 \mu+1 \leq m$. The solution of system (14) exists for any $m \in \mathbb{N}$ but is nonunique for $m \geq 3$. A reasonable solution of system (14) can be determined with the help of the Wiener inversion of $\mathfrak{B}_{m}$. The Wiener theorem in the Laurent series formulation states the following (see [11]): if $b_{k} \in \mathbb{C}, k \in \mathbb{Z}$, satisfy

$$
\sum_{k \in \mathbb{Z}}\left|b_{k}\right|<\infty, \quad b(z):=\sum_{k \in \mathbb{Z}} b_{k} z^{k} \neq 0 \quad \text { for all } z \in \mathbb{C} \text { with }|z|=1,
$$

then also the function $a(z):=\frac{1}{b(z)}$ has an expansion $a(z)=\sum_{k \in \mathbb{Z}} a_{k} z^{k}$ with $\sum_{k \in \mathbb{Z}}\left|a_{k}\right|<\infty$. The Toeplitz matrix $\mathfrak{A}=\left(a_{k-j}\right)_{k, j \in \mathbb{Z}}$ is the (Wiener) inverse to $\mathfrak{B}=\left(b_{k-j}\right)_{k, j \in \mathbb{Z}}$, namely, $\mathfrak{B A f}=\mathfrak{A} \mathfrak{B} \mathbf{f}=\mathbf{f}$ for any bounded bisequence $\mathbf{f}=\left(f_{j}\right)$.

It is possible to find the Wiener inverse $\mathfrak{A}_{m}=\left(a_{k-j, m}\right)$ of $\mathfrak{B}_{m}=\left(b_{k-j, m}\right)$ defined in (15), and then (12) with $d_{k}=\sum_{j \in \mathbb{Z}} a_{k-j, m} f_{j}, k \in \mathbb{Z}$, determines an interpolant that we denote by $Q_{h, m} f$ and call the Wiener interpolant. Namely, introducing the polynomial $P_{m} \in \mathcal{P}_{2 \mu}$ by

$$
P_{m}(z)=\sum_{|k| \leq \mu} b_{k, m} z^{k+\mu}
$$

it occurs [8] that $P_{m}$ has $\mu$ simple roots $z_{\nu, m}, \nu=1, \ldots, \mu$, in the interval $(-1,0)$, and the remaining $\mu$ roots are of the form $\frac{1}{z_{\nu, m}} \in(-\infty,-1)$. Hence for $b_{m}(z)=\sum_{k} b_{k, m} z^{k}$, conditions (16) are satisfied. Moreover, it occurs that

$$
a_{k, m}=\sum_{\nu=1}^{\mu} \frac{z_{\nu, m}^{\mu-1}}{P_{m}^{\prime}\left(z_{\nu, m}\right)} z_{\nu, m}^{|k|}, k \in \mathbb{Z}
$$


and $\sum_{k \in \mathbb{Z}} a_{k, m}=1, \sum_{k \in \mathbb{Z}}\left|a_{k, m}\right|=\frac{(-1)^{\mu}}{P_{m}(-1)}, a_{k, m}=(-1)^{k}\left|a_{k, m}\right|, k \in \mathbb{Z}$. We see that $a_{k, m}, k \in \mathbb{Z}$, decay exponentially as $|k| \rightarrow \infty$ and are of alternating sign. Thus the cardinal interpolation process is reduced to the finding of the roots of the (characteristic) polynomial $P_{m} \in \mathcal{P}_{2 \mu}$ defined in (17).

The following fundamental result holds true.

Theorem $2.1([4-6,8])$. For $f \in B C(\mathbb{R})$, the Wiener interpolant

$$
\left(Q_{h, m} f\right)(x)=\sum_{k \in \mathbb{Z}}\left(\sum_{j \in \mathbb{Z}} a_{k-j, m} f\left(\left(j+\frac{m}{2}\right) h\right)\right) B_{m}\left(h^{-1} x-k\right), \quad x \in \mathbb{R},
$$

with $a_{k, m}$ defined in (18) is the only bounded interpolant (12)-(13); $Q_{h, m} f$ is well defined also for any $f \in C(\mathbb{R})$ of at most polynomial growth as $|x| \rightarrow \infty$, and it is the only at most polynomially growing interpolant (12)-(13).

In the vector space of all bisequences $\left(d_{j}\right)_{j \in \mathbb{Z}}$, the null space $\mathcal{N}\left(\mathfrak{B}_{m}\right)$ of $\mathfrak{B}_{\mathfrak{m}}=$ $\left(b_{k-j, m}\right)_{k, j \in \mathbb{Z}}$ is of dimension $2 \mu$ being spanned by the bisequences $\left(z_{\nu, m}^{j}\right)_{j \in \mathbb{Z}}$, $\left(z_{\nu, m}^{-j}\right)_{j \in \mathbb{Z}}, \nu=1, \ldots, \mu$. Thus, $\left|d_{j}^{(0)}\right| \rightarrow \infty$ exponentially as $j \rightarrow \infty$ or $j \rightarrow-\infty$ for any nontrivial $\left(d_{j}^{(0)}\right) \in \mathcal{N}\left(\mathfrak{B}_{m}\right)$.

It holds $\left(Q_{h, m} f\right)(x)=\sum_{j \in \mathbb{Z}} f\left(\left(j+\frac{m}{2}\right) h\right) F_{m}\left(h^{-1} x+j\right)$, where $F_{m}(x):=$ $\sum_{k \in \mathbb{Z}} a_{k, m} B_{m}(x-k), x \in \mathbb{R}$, is the fundamental spline, $F_{m}\left(j+\frac{m}{2}\right)=\delta_{j, 0}, j \in \mathbb{Z}$, $\delta_{j, k}$ is the Kronecker symbol. This representation form of $Q_{h, m} f$ implies the equality

$$
\left\|Q_{h, m}\right\|_{B C(\mathbb{R}) \rightarrow B C(\mathbb{R})}=\sup _{x \in \mathbb{R}} \sum_{j \in \mathbb{Z}}\left|F_{m}(x+j)\right|=\max _{x \in\left[\frac{m}{2}, \frac{m+1}{2}\right]} \sum_{j \in \mathbb{Z}}\left|F_{m}(x+j)\right|
$$

where we took into account that the function $\varphi_{m}(x):=\sum_{j \in \mathbb{Z}}\left|F_{m}(x+j)\right|$ is 1 -periodic, and due to (9) $\varphi_{m}\left(\frac{m}{2}-x\right)=\varphi_{m}\left(\frac{m}{2}+x\right), x \in \mathbb{R}$. The numerical values of $q_{m}:=\left\|Q_{h, m}\right\|_{B C(\mathbb{R}) \rightarrow B C(\mathbb{R})}$ and $\alpha_{m}:=\sum_{k \in \mathbb{Z}}\left|a_{k, m}\right|$ for some $m$ are presented in the following table originating from [3]:

\begin{tabular}{cccccccccc}
$m$ & 3 & 4 & 5 & 6 & 7 & 8 & 9 & 10 & 20 \\
\hline$q_{m}$ & 1.414 & 1.549 & 1.706 & 1.816 & 1.916 & 2.000 & 2.075 & 2.142 & 2.583 \\
$\alpha_{m}$ & 2.000 & 3.000 & 4.800 & 7.500 & 11.80 & 18.53 & 29.11 & 45.73 & 4182
\end{tabular}

We can observe that $\frac{\alpha_{m+1}}{\alpha_{m}} \rightarrow \frac{\pi}{2}=1.5707963268 \ldots$ as $m \rightarrow \infty$; for $m=20$ this ratio is 1.570796327 . For $4 \leq m \leq 20$, the computed values of $q_{m}$ fit into the model $q_{m} \leq \frac{e}{4}+\frac{2}{\pi} \log m$, and possibly $q_{m}-\left(\frac{e}{4}+\frac{2}{\pi} \log m\right) \rightarrow 0$ as $m \rightarrow \infty$; for $m=20$ this difference is of order 0.003. It is challenging to confirm these empiric guesses analytically (or disprove them). 
2.3. Euler perfect splines. A spline $E \in S_{h, m}$ is called perfect if $E^{(m-1)}(x)=$ $(-1)^{i}$ for $h<x<(i+1) h, i \in \mathbb{Z}$. Elementary claims listed below are often used in the theory of splines, see $[2,4-6]$.

For $m=1$, the Euler perfect spline $E_{h, 1} \in S_{h, 1}$ is given by the formula

$$
E_{h, 1}(x)=\operatorname{sign} \sin \left(h^{-1} \pi x\right)=\frac{4}{\pi} \sum_{k=0}^{\infty} \frac{\sin \left((2 k+1) h^{-1} \pi x\right)}{2 k+1} ;
$$

it is a piecewise constant $2 h$-periodic function and $\int_{0}^{2 h} E_{h, 1}(x) d x=0$. For $m \geq 2$, the Euler perfect spline $E_{h, m} \in S_{h, m}$ is determined recursively as

$$
E_{h, m}(x)= \begin{cases}\int_{\frac{h}{2}}^{x} E_{h, m-1}(y) d y, & m=2 l \\ \int_{0}^{x} E_{h, m-1}(y) d y, & m=2 l+1\end{cases}
$$

the lower bounds of integration are chosen so that the $2 h$-periodicity and the zero mean value of $E_{h, m-1}$ over the period are inherited to $E_{h, m}$. Starting from (20), this recursively implies that, for $m \geq 2$,

$$
E_{h, m}(x)=\left\{\begin{array}{l}
\frac{4}{\pi} \frac{(-1)^{l} h^{m-1}}{\pi^{m-1}} \sum_{k=0}^{\infty} \frac{\cos \left((2 k+1) h^{-1} \pi x\right)}{(2 k+1)^{m}}, m=2 l \\
\frac{4}{\pi} \frac{(-1)^{l} h^{m-1}}{\pi^{m-1}} \sum_{k=0}^{\infty} \frac{\sin \left((2 k+1) h^{-1} \pi x\right)}{(2 k+1)^{m}}, m=2 l+1 .
\end{array}\right.
$$

In particular, $E_{h, 2}$ is a continuous piecewise linear function with the knot values $E_{h, 2}(i h)=(-1)^{i+1} \frac{h}{2}, i \in \mathbb{Z}$; a consequence used in Section 3 is that

$$
E_{h, 2}\left(x_{1}\right)-E_{h, 2}\left(x_{2}\right)=(-1)^{i}\left(x_{1}-x_{2}\right), \quad \text { for } x_{1}, x_{2} \in(i h,(i+1) h), i \in \mathbb{Z} .
$$

By construction, $E_{h, m}^{\prime}=E_{h, m-1}$ for $m \geq 2$. It follows from (21) that $x=\left(i+\frac{1}{2}\right) h, i \in \mathbb{Z}$, are the zeroes of $E_{h, m}$ for even $m$, and $x=i h, i \in \mathbb{Z}$, are the zeroes of $E_{h, m}$ for odd $m$. A unified formulation is that $x=\left(i+\frac{m-1}{2}\right) h$, $i \in \mathbb{Z}$, are the zeroes of $E_{h, m}$ and $x=\left(i+\frac{m}{2}\right) h, i \in \mathbb{Z}$, are the local extrema of $E_{h, m}$ (the zeroes of $E_{h, m}^{\prime}=E_{h, m-1}$ ). There are no other zeroes and extrema of $E_{h, m}$ - this can be seen recursively using Rolle's theorem. It is clear also that the zeroes of $E_{h, m}$ are simple. Further,

$$
\left\|E_{h, m}\right\|_{\infty}=\Phi_{m} \pi^{-(m-1)} h^{m-1}, \quad m \in \mathbb{N},
$$

where

$$
\Phi_{m}=\frac{4}{\pi}\left\{\begin{array}{ll}
\sum_{k=0}^{\infty} \frac{1}{(2 k+1)^{m}}, & m=2 l \\
\sum_{k=0}^{\infty} \frac{(-1)^{k}}{(2 k+1)^{m}}, & m=2 l+1
\end{array}\right\}=\frac{4}{\pi} \sum_{k=0}^{\infty} \frac{(-1)^{k m}}{(2 k+1)^{m}}, \quad m \in \mathbb{N},
$$


is known as the Favard constant. In particular, $\Phi_{1}=1, \Phi_{2}=\frac{\pi}{2}, \Phi_{3}=\frac{\pi^{2}}{8}$, $\Phi_{4}=\frac{\pi^{3}}{24}$, and it holds $\lim _{m \rightarrow \infty} \Phi_{m}=\frac{4}{\pi}, \Phi_{1}<\Phi_{3}<\Phi_{5}<\cdots<\frac{4}{\pi}<\cdots<\Phi_{6}<$ $\Phi_{4}<\Phi_{2}$.

\section{The main result}

Due to (3), a function $f \in V^{m, \infty}(\mathbb{R})$ is at most polynomially growing as $|x| \rightarrow \infty$, hence the Wiener interpolant $Q_{h, m} f$ is well defined for it. For $f \in V^{m, \infty}(\mathbb{R})$, it holds

$$
\left|f^{(m-1)}\left(x_{1}\right)-f^{(m-1)}\left(x_{2}\right)\right| \leq\left\|f^{(m)}\right\|_{\infty}\left|x_{1}-x_{2}\right|, \quad x_{1}, x_{2} \in \mathbb{R} .
$$

We are ready to prove the main result of the paper.

Theorem 3.1. For $f \in V^{m, \infty}(\mathbb{R}), m \in \mathbb{N}$, there hold the pointwise estimate

$$
\left|f(x)-\left(Q_{h, m} f\right)(x)\right| \leq\left\|f^{(m)}\right\|_{\infty}\left|E_{h, m+1}(x)\right|, \quad x \in \mathbb{R},
$$

and the uniform estimate

$$
\left\|f-Q_{h, m} f\right\|_{\infty} \leq \Phi_{m+1} \pi^{-m} h^{m}\left\|f^{(m)}\right\|_{\infty} .
$$

For $f=E_{h, m+1} \in W^{m, \infty}(\mathbb{R})$, inequalities (26) and (27) turn into equalities.

Proof. (i) Proof scheme. First of all we note that the last assertion concerning the sharpness of estimates (26) and (27) is elementary. Indeed, $E_{h, m+1}$ vanishes at $\left(i+\frac{m}{2}\right) h, i \in \mathbb{Z}$, which are the interpolation points for $Q_{h, m}$, hence $Q_{h, m} E_{h, m+1}=0$; further, $\left\|E_{h, m+1}^{(m)}\right\|_{\infty}=\left\|E_{h, 1}\right\|_{\infty}=1$. Thus for $f=E_{h, m+1}$ (26) turns into the equality $\left|E_{h, m+1}(x)\right|=\left|E_{h, m+1}(x)\right|$, whereas (27) turns into the equality $\left\|E_{h, m+1}\right\|_{\infty}=\Phi_{m+1} \pi^{-m} h^{m}$, cf. (23).

Estimate (27) immediately follows from (26) and (23). So it remains to establish (26) only. For $m=1$ and $m=2$, usual local estimates of piecewise constant and piecewise linear interpolant can be presented in the form (26) remembering that $\Phi_{2}=\frac{\pi}{2}, \Phi_{3}=\frac{\pi^{2}}{8}$. So we may assume that $m \geq 3$. We prove (26) during four stages: in (ii) for periodic $f \in W^{m, \infty}(\mathbb{R})$, in (iii) for compactly supported $f \in W^{m, \infty}(\mathbb{R})$, in (iv) for $f \in V^{m, \infty}(\mathbb{R})$ with a special growth estimate, and in $(\mathrm{v})$ for arbitrary $f \in V^{m, \infty}(\mathbb{R})$. Technically, the proof parts (iii)-(v) are based on Lemma 3.2 formulated and proved after the proof part (ii).

(ii) Periodic case (cf. [2]). Here we prove (26) for $f \in W^{m, \infty}(\mathbb{R})$ which is periodic with a period $p=2 n h, n \in \mathbb{N}, p \in \mathbb{R}$. Then also $Q_{h, m} f$ is $p$ periodic, and so is $E_{h, m+1}$ (recall that $E_{h, m+1}$ has the period $2 h$ ). We show that the violation of (26) for such $f$ involves a contradiction. Let $\xi \in[0, p)$ be a point where (26) is violated: $\left|f(\xi)-\left(Q_{h, m} f\right)(\xi)\right|>\left\|f^{(m)}\right\|_{\infty}\left|E_{h, m+1}(\xi)\right|$; clearly 
$\xi \neq\left(i+\frac{m}{2}\right) h, i \in \mathbb{Z}$, since $f-Q_{h, m} f$ vanishes at those points. Take $\theta \in \mathbb{R}$, $|\theta|<1$, such that $\theta\left(f(\xi)-\left(Q_{h, m} f\right)(\xi)\right)=\left\|f^{(m)}\right\|_{\infty} E_{h, m+1}(\xi)$, and introduce the $p$-periodic function

$$
g=\left\|f^{(m)}\right\|_{\infty} E_{h, m+1}-\theta\left(f-Q_{h, m} f\right) \in C^{m-2}(\mathbb{R}) .
$$

In the period interval $[0, p), g$ has at least $2 n+1$ zeroes, namely $\xi$ and $2 n$ interpolation points $\left(i+\frac{m}{2}\right) h, 0 \leq i+\frac{m}{2}<2 n$. It is easily seen that if $a$ continuous p-periodic function $u$ has $l$ zeroes in $[0, p)$, then it has at least $l$ local extreme points in $[0, p)$ (claim 1); of course, those are zeroes of $u^{\prime}$ if $u$ is differentiable. Applying claim 1 recursively to $g$ and its derivatives we conclude that $v:=g^{(m-2)} \in C(\mathbb{R})$ has at least $2 n+1$ (local) extreme points in $[0, p)$. But next we show that actually $v$ has at most $2 n$ extreme points in $[0, p)$ (claim 2 ) and thus we have the desired contradiction. Indeed, for $x_{1}, x_{2} \in(i h,(i+1) h)$,

$$
v^{\prime}\left(x_{1}\right)-v^{\prime}\left(x_{2}\right)=\left\|f^{(m)}\right\|_{\infty}\left(E_{h, 2}\left(x_{1}\right)-E_{h, 2}\left(x_{2}\right)\right)-\theta\left(f^{(m-1}\left(x_{1}\right)-f^{(m-1)}\left(x_{2}\right)\right)
$$

since $\left(Q_{h, m} f\right)^{(m-1)}(x)$ is a constant for $x \in(i h,(i+1) h)$. Due to $(22)$ and $(25)$, for $x_{1}, x_{2} \in(i h,(i+1) h)$,

$$
v^{\prime}\left(x_{1}\right)-v^{\prime}\left(x_{2}\right) \begin{cases}\geq(1-|\theta|)\left\|f^{(m)}\right\|_{\infty}\left(x_{1}-x_{2}\right) & \text { if } i \text { is even } \\ \leq(-1+|\theta|)\left\|f^{(m)}\right\|_{\infty}\left(x_{1}-x_{2}\right) & \text { if } i \text { is odd }\end{cases}
$$

We may assume that $f$ is not identically constant since in the case of constant $f$ (26) holds trivially. Then due to periodicity, $\left\|f^{(m)}\right\|_{\infty}>0$, and $v^{\prime}$ is in the interval $(i h,(i+1) h)$ strictly increasing for even $i$ and strictly decreasing for odd $i$. Hence, inside an interval $(i h,(i+1) h), v$ may have at most one extreme point. Clearly, $v$ has the following further properties (claim 3):

$\star$ if a knot $i h, i \in \mathbb{Z}$, is a minimum (respectively, maximum) point of $v$ then this one of the adjacent intervals $((i-1) h, i h)$ and $(i h,(i+1) h)$ on which $v^{\prime}$ increases (respectively, decreases), is free from extreme points of $v$;

$\star$ for an interval $(j h,(j+1) h), j \in \mathbb{Z}$, on which $v^{\prime}$ increases (respectively, decreases), at least one of the end points $j h$ and $(j+1) h$ is not a minimum point (respectively, a maximum point) of $v$.

Denote by $\mathcal{E}$ the set of extreme points of $v$ in the period interval $[0, p)$ and by $\mathcal{G}$ the set of intervals $(i h,(i+1) h), i=0, \ldots, 2 n-1$. Define a mapping $\mu: \mathcal{E} \rightarrow \mathcal{G}$ by the following rules:

$\star$ if $x \in \mathcal{E}$ belongs to an interval $(i h,(i+1) h)$, then $\mu(x)=(i h,(i+1) h)$;

$\star$ if $x=i h \in \mathcal{E}, 1 \leq i \leq 2 n-1$, and $x$ is a minimum (respectively, maximum) point of $v$, then $\mu(x)$ is either $((i-1) h, i h)$ or $(i h,(i+1) h)$, namely, $\mu(x)$ is this one of these two candidates-intervals on which $v^{\prime}$ is increasing (respectively, decreasing); 
$\star$ if $0 \in \mathcal{E}$ (by periodicity, then also $p=2 n h$ is an extreme point of $v$ but not in $\mathcal{E})$, the choice is made by the same rule between the candidates $((2 n-1) h, 2 n h)$ and $(0, h)$.

Due to claim 3, the mapping $\mu: \mathcal{E} \rightarrow \mathcal{G}$ is injective, i.e., for $x, x^{\prime} \in \mathcal{E}$, $x \neq x^{\prime}$, there holds $\mu(x) \neq \mu\left(x^{\prime}\right)$. Hence, for the cardinalities of sets $\mathcal{E}$ and $\mathcal{G}$ we have $\operatorname{card}(\mathcal{E}) \leq \operatorname{card}(\mathcal{G})=2 n$. This proves claim 2 and completes the proof of Theorem 3.1 in the periodic case.

Now we interrupt the proof of Theorem 3.1 in order to establish an auxiliary result that we need to continue the proof. It concerns the pointwise convergence of interpolants for functions depending on a parameter.

Lemma 3.2. Suppose that for functions $g_{\delta} \in C(\mathbb{R}), \delta>0$, we have

$$
\begin{aligned}
g_{\delta}(x) & \rightarrow 0 & & \text { as } \delta \rightarrow 0 \text { for every } x \in \mathbb{R} \\
\left|g_{\delta}(x)\right| & \leq c\left(1+|x|^{r}\right), & & x \in \mathbb{R},
\end{aligned}
$$

where $c \geq 0$ and $r \geq 0$ are independent of $\delta$. Then also

$$
\left(Q_{h, m} g_{\delta}\right)(x) \rightarrow 0 \quad \text { as } \delta \rightarrow 0 \text { for every } x \in \mathbb{R}
$$

Proof. Fix an arbitrary $x \in \mathbb{R}$ and take $i \in \mathbb{Z}$ such that $x \in[i h,(i+1) h)$. Then (see (19) and (11))

$$
\begin{aligned}
\left(Q_{h, m} g_{\delta}\right)(x) & =\sum_{k=i-m+1}^{i}\left(\sum_{j \in \mathbb{Z}} a_{j, m} g_{\delta}\left(\left(k-j+\frac{m}{2}\right) h\right)\right) B_{m}\left(h^{-1} x-k\right) \\
\left|\left(Q_{h, m} g_{\delta}\right)(x)\right| & \leq \max _{i-m+1 \leq k \leq i} \sum_{j \in \mathbb{Z}}\left|a_{j, m}\right|\left|g_{\delta}\left(\left(k-j+\frac{m}{2}\right) h\right)\right| .
\end{aligned}
$$

It is sufficient to show that $\sum_{j \in \mathbb{Z}}\left|a_{j, m}\right|\left|g_{\delta}\left(\left(k-j+\frac{m}{2}\right) h\right)\right| \rightarrow 0$ as $\delta \rightarrow 0$ for fixed $k \in \mathbb{Z}$. Fixing an $\varepsilon>0$, represent $\sum_{j \in \mathbb{Z}}=\sum_{|j| \leq N}+\sum_{|j|>N}$ with $N=N(\varepsilon, k)$ so large that $\sum_{|j|>N}\left|a_{j, m}\right|\left(1+\left(|j-k|+\frac{m}{2}\right)^{r} h^{r}\right) \leq \varepsilon$; such $N$ exists since $a_{j, m}$ decays exponentially as $|j| \rightarrow \infty$. Using (29) we obtain

$$
\sum_{|j|>N}\left|a_{j, m}\right|\left|g_{\delta}\left(\left(k-j+\frac{m}{2}\right) h\right)\right| \leq c \sum_{|j|>N}\left|a_{j, m}\right|\left(1+\left(|j-k|+\frac{m}{2}\right)^{r} h^{r}\right) \leq c \varepsilon .
$$

Due to (28), for sufficiently small $\delta>0$, we have

$$
\sum_{|j| \leq N}\left|a_{j, m}\right|\left|g_{\delta}\left(\left(i-j+\frac{m}{2}\right) h\right)\right|<\varepsilon, \quad \sum_{j \in \mathbb{Z}}\left|a_{j, m}\right|\left|g_{\delta}\left(\left(i-j+\frac{m}{2}\right) h\right)\right|<(c+1) \varepsilon
$$

that completes the proof of (30). 
Continuation of the proof of Theorem 3.1. (iii) Case of compactly supported $f$. Next we prove (26) for functions $f \in W^{m, \infty}(\mathbb{R})$ having a compact support. Assume that $f(x)=0$ for $x \geq \rho$ where $\rho>0$. Take a number $p=2 n h$ with $n \in \mathbb{N}$ such that $p>2 \rho$, and introduce the function $f_{p}(x)=\sum_{k \in \mathbb{Z}} f(x+k p)$ (for a fixed $x$, this series contains at most one nonzero term). The function $f_{p}$ is $p$-periodic and still $f_{p} \in W^{m, \infty}(\mathbb{R}),\left\|f_{p}^{(m)}\right\|_{\infty}=\left\|f^{(m)}\right\|_{\infty}$. Represent

$$
f-Q_{h, m} f=f_{p}-Q_{h, m} f_{p}+\left(I-Q_{h, m}\right)\left(f-f_{p}\right) .
$$

As proved in (ii), (26) holds true for $f_{p}$ : for any $x \in \mathbb{R}$,

$$
\left|f_{p}(x)-\left(Q_{h, m} f_{p}\right)(x)\right| \leq\left\|f_{p}^{(m)}\right\|_{\infty}\left|E_{h, m+1}(x)\right|=\left\|f^{(m)}\right\|_{\infty}\left|E_{h, m+1}(x)\right| .
$$

To establish (26) for $f$, it now suffices to show that for any fixed $x \in \mathbb{R}$, $\left(\left(I-Q_{h, m}\right)\left(f-f_{p}\right)\right)(x) \rightarrow 0$ as $p \rightarrow \infty$. Clearly, $g_{p}(x):=f(x)-f_{p}(x) \rightarrow 0$ as $p \rightarrow \infty$ for fixed $x \in \mathbb{R}\left(g_{p}(x)=0\right.$ for sufficiently large $\left.p\right)$, so it remains to observe that by Lemma 3.2 (with $\delta=\frac{1}{p}$ ) also $\left(Q_{h, m} g_{p}\right)(x) \rightarrow 0$ as $p \rightarrow \infty$.

(iv) Case of $f \in V^{m, \infty}(\mathbb{R})$ of restricted growth. Now we extend estimate (26) to $f \in V^{m, \infty}(\mathbb{R})$ satisfying the condition

$$
\frac{f^{(k)}(x)}{x^{m-k}} \rightarrow 0 \quad \text { as }|x| \rightarrow \infty, k=0, \ldots, m-1,
$$

which elementarily implies that

$$
\delta^{m-k} \sup _{|x| \leq 1 / \delta}\left|f^{(k)}(x)\right| \rightarrow 0 \quad \text { as } 0<\delta \rightarrow 0, k=0, \ldots, m-1 .
$$

Take a "cutting" function $e \in C^{m}(\mathbb{R})$ such that $0 \leq e(x) \leq 1$ for all $x \in \mathbb{R}$, $e(x)=1$ for $|x| \leq \frac{1}{2}, e(x)=0$ for $|x| \geq 1$. Denote $f_{\delta}(x)=e(\delta x) f(x)$ and represent

$$
f(x)-\left(Q_{h, m} f\right)(x)=f_{\delta}(x)-\left(Q_{h, m} f_{\delta}\right)(x)+\left(\left(I-Q_{h, m}\right)\left(f-f_{\delta}\right)\right)(x) .
$$

Clearly $f_{\delta} \in W^{m, \infty}(\mathbb{R})$ and $\operatorname{supp} f_{\delta} \subset\left[-\frac{1}{\delta}, \frac{1}{\delta}\right]$. As proven in (iii), (26) holds for $f_{\delta}$ :

$$
\left|f_{\delta}(x)-\left(Q_{h, m} f_{\delta}\right)(x)\right| \leq\left\|f_{\delta}^{(m)}\right\|_{\infty}\left|E_{h, m+1}(x)\right|, \quad x \in \mathbb{R} .
$$

Denoting $c_{k}=\max _{x \in \mathbb{R}}\left|e^{(k)}(x)\right|$, we have due to (32)

$$
\begin{aligned}
\left\|f_{\delta}^{(m)}\right\|_{\infty} & \leq \sum_{k=0}^{m}\left(\begin{array}{c}
m \\
k
\end{array}\right) \delta^{m-k} \sup _{x \in \mathbb{R}}\left|e^{(m-k)}(\delta x) \| f^{(k)}(x)\right| \\
& \leq\left\|f^{(m)}\right\|_{\infty}+\sum_{k=0}^{m-1}\left(\begin{array}{c}
m \\
k
\end{array}\right) c_{m-k} \delta^{m-k} \sup _{|x| \leq \frac{1}{\delta}}\left|f^{(k)}(x)\right| \rightarrow\left\|f^{(m)}\right\|_{\infty} \quad \text { as } \delta \rightarrow 0 .
\end{aligned}
$$


Further, $g_{\delta}(x):=f(x)-f_{\delta}(x)=0$ for a fixed $x \in \mathbb{R}$ and sufficiently small $\delta>0$, and by condition (31) $\left|g_{\delta}(x)\right| \leq c\left(1+|x|^{m}\right), x \in \mathbb{R}$. Due to Lemma 3.2, $\left(Q_{h, m}\left(f-f_{\delta}\right)\right)(x) \rightarrow 0$ as $\delta \rightarrow 0$ for any $x \in \mathbb{R}$. With these considerations, (26) for $f$ follows from (33) as $\delta \rightarrow 0$.

(v) Case of arbitrary $f \in V^{m, \infty}(\mathbb{R})$. Starting from the Taylor formula (2), introduce the approximation

$$
f_{\delta}(x):=\sum_{l=0}^{m-1} \frac{f^{(l)}(0)}{l !} x^{l}+\frac{1}{(m-1) !} \int_{0}^{x}(x-t)^{m-1} \theta(\delta t) f^{(m)}(t) d t, \quad x \in \mathbb{R}, \delta>0,
$$

where $\theta(x)=1$ for $|x| \leq 1$ and $\theta(x)=0$ for $|x|>1$. For any $\delta>0$, $f_{\delta} \in V^{m, \infty}(\mathbb{R})$ and $f_{\delta}$ satisfies (31). Further, $\left\|f_{\delta}^{(m)}\right\|_{\infty} \leq\left\|f^{(m)}\right\|_{\infty}$ for $\delta>0$ and $f_{\delta}(x)=f(x)$ for a fixed $x \in \mathbb{R}$ if $\delta>0$ is sufficiently small. With this $f_{\delta}$, we have the equality (33) in which, due to (iv),

$$
\left|f_{\delta}(x)-\left(Q_{h, m} f_{\delta}\right)(x)\right| \leq\left\|f_{\delta}^{(m)}\right\|_{\infty}\left|E_{h, m+1}(x)\right| \leq\left\|f^{(m)}\right\|_{\infty}\left|E_{h, m+1}(x)\right|, \quad x \in \mathbb{R} .
$$

Clearly, $|f(x)|+\left|f_{\delta}(x)\right| \leq c\left(1+|x|^{m}\right)$ for $x \in \mathbb{R}$. Using Lemma 3.2 we obtain that $\left(Q_{h, m}\left(f-f_{\delta}\right)\right)(x) \rightarrow 0$ as $\delta \rightarrow 0$, and (26) for $f$ follows from (33) as $\delta \rightarrow 0$. The proof of Theorem 3.1 is complete.

Remark 3.3. Using the Banach-Steinhaus theorem and Theorem 3.1, it is easily seen that $\left\|f-Q_{h, m} f\right\|_{\infty} \rightarrow 0$ as $h \rightarrow 0$ for any $f \in B U C(\mathbb{R})$.

\section{Optimality of the spline interpolation}

Let us discuss optimality properties of the spline interpolation compared with other approximation methods that use the same information about a given function $f$ as the interpolant $Q_{h, m} f$ - the values of $f$ on the uniform grid $\mathbb{Z}_{h, m}=\left\{\left(j+\frac{m}{2}\right) h: j \in \mathbb{Z}\right\}$. Such a method can be identified with a mapping $M_{h}: C\left(\mathbb{Z}_{h, m}\right) \rightarrow C(\mathbb{R})$, where $C\left(\mathbb{Z}_{h, m}\right)$ is the vector space of grid functions defined on $\mathbb{Z}_{h}$.

Theorem 4.1. For given $\gamma>0$, we have in accordance to Theorem 3.1

$$
\sup _{f \in V^{m, \infty}(\mathbb{R}),\left\|f^{(m)}\right\|_{\infty} \leq \gamma}\left\|f-Q_{h, m} f\right\|_{\infty}=\Phi_{m+1} \pi^{-m} h^{m} \gamma
$$

whereas for any mapping $M_{h}: C\left(\mathbb{Z}_{h, m}\right) \rightarrow C(\mathbb{R})$ (linear or nonlinear, continuous or discontinuous), it holds

$$
\sup _{f \in W^{m, \infty}(\mathbb{R}),\left\|f^{(m)}\right\|_{\infty} \leq \gamma}\left\|f-M_{h}\left(\left.f\right|_{\mathbb{Z}_{h, m}}\right)\right\|_{\infty} \geq \Phi_{m+1} \pi^{-m} h^{m} \gamma
$$


Proof. If $M_{h}(\mathbf{0}) \notin B C(\mathbb{R}),(34)$ is trivially fulfilled. So we may assume that $M_{h}(\mathbf{0}) \in B C(\mathbb{R})$. Consider two functions $f_{ \pm}= \pm \gamma E_{h, m+1}$. Clearly, $f_{ \pm} \in$ $W^{m, \infty}(\mathbb{R}),\left\|f_{ \pm}^{(m)}\right\|_{\infty}=\gamma$, and since $\left.E_{h, m+1}\right|_{\mathbb{Z}_{h}}=\mathbf{0}$, we obtain (34) by the following argument:

$$
\begin{aligned}
& \sup \left\{\left\|f-M_{h}\left(\left.f\right|_{\mathbb{Z}_{h, m}}\right)\right\|_{\infty}: f \in W^{m, \infty}(\mathbb{R}),\left\|f^{(m)}\right\|_{\infty} \leq \gamma\right\} \\
& \geq \max \left\{\left\|f_{+}-M_{h}\left(\left.f_{+}\right|_{\mathbb{Z}_{h, m}}\right)\right\|_{\infty},\left\|f_{-}-M_{h}\left(\left.f_{-}\right|_{\mathbb{Z}_{h, m}}\right)\right\|_{\infty}\right\} \\
& =\max \left\{\left\|f_{+}-M_{h}(\mathbf{0})\right\|_{\infty},\left\|f_{-}-M_{h}(\mathbf{0})\right\|_{\infty}\right\} \\
& \geq \frac{1}{2}\left(\left\|f_{+}-M_{h}(\mathbf{0})\right\|_{\infty}+\left\|f_{-}-M_{h}(\mathbf{0})\right\|_{\infty}\right) \\
& \geq \frac{1}{2}\left\|f_{+}-f_{-}\right\|_{\infty}
\end{aligned}
$$

and $\frac{1}{2}\left\|f_{+}-f_{-}\right\|_{\infty}=\left\|E_{h, m+1}\right\|_{\infty} \gamma=\Phi_{m+1} \pi^{-m} h^{m} \gamma$.

Remark 4.2. For functions with compact supports, similar result as (34) holds asymptotically as $h \rightarrow 0$. Denote by $W_{[0,1]}^{m, \infty}(\mathbb{R})$ the subspace of $W^{m, \infty}(\mathbb{R})$ consisting of functions $f \in W^{m, \infty}(\mathbb{R})$ with supports in $[0,1]$. For any mapping $M_{h}: C\left(\mathbb{Z}_{h, m}\right) \rightarrow C(\mathbb{R})$, it holds

$$
\liminf _{h \rightarrow 0} \sup _{f \in W_{[0,1]}^{m, \infty}(\mathbb{R}),\left\|f^{(m)}\right\|_{\infty} \leq \gamma} \frac{\left\|f-M_{h}\left(\left.f\right|_{\mathbb{Z}_{h, m}}\right)\right\|_{\infty}}{\Phi_{m+1} \pi^{-m} h^{m} \gamma} \geq 1 .
$$

This follows by a slight modification of the argument in the proof of Theorem 4.1. Namely, instead of $f_{ \pm}= \pm \gamma E_{h, m+1}$, use $f_{ \pm}= \pm \gamma e E_{h, m+1}$ where $e \in C^{m}(\mathbb{R})$ is supported in $(0,1), 0 \leq e(x) \leq 1$ for all $x \in \mathbb{R}$ and $e(x)=1$ for $\frac{1}{3} \leq$ $x \leq \frac{2}{3}$. Then for sufficiently small $h$, it still holds $\left\|e E_{h, m+1}\right\|_{\infty}=\left\|E_{h, m+1}\right\|_{\infty}=$ $\Phi_{m+1} \pi^{-m} h^{m}$, and the Leibniz differentiation rule yields $\left\|\left(e E_{h, m+1}\right)^{(m)}\right\|_{\infty} \rightarrow 1$ as $h \rightarrow 0$.

Remark 4.3. Let $n \in \mathbb{N}$ be even and $h=\frac{1}{n}$. Consider the subspace $C_{\text {per }}(\mathbb{R})$ of $C(\mathbb{R})$ consisting of 1 -periodic continuous functions on $\mathbb{R}$, and denote $W_{\text {per }}^{m, \infty}(\mathbb{R})=$ $C_{\text {per }}(\mathbb{R}) \cap W^{m, \infty}(\mathbb{R})$; denote by $C_{\text {per }}\left(\mathbb{Z}_{h}\right)$ the space of 1 -periodic (grid) functions on the grid $\mathbb{Z}_{h}$, i.e., $f_{h}\left(\left(i+\frac{m}{2}\right) h\right)=f_{h}\left(1+\left(i+\frac{m}{2}\right) h\right), i \in \mathbb{Z}$, for $f_{h} \in C_{\text {per }}\left(\mathbb{Z}_{h}\right)$. Then for any mapping $M_{h}: C_{\text {per }}\left(\mathbb{Z}_{h}\right) \rightarrow C_{\text {per }}(\mathbb{R})$, it holds

$$
\sup _{f \in W_{\mathrm{per}}^{m, \infty}(\mathbb{R}),\left\|f^{(m)}\right\|_{\infty} \leq \gamma}\left\|f-M_{h}\left(\left.f\right|_{\mathbb{Z}_{h}}\right)\right\|_{\infty} \geq \Phi_{m+1} \pi^{-m} h^{m} \gamma
$$

The proof is same as in the case of Theorem 4.1, we only need to observe that $E_{h, m+1} \in W_{\text {per }}^{m, \infty}(\mathbb{R})$ for even $n$. 


\section{Error estimates for derivatives}

To derive error estimates for the derivatives of the spline interpolant (Theorem 5.3), we first establish some technical results (Lemma 5.1 and Lemma 5.2).

Lemma 5.1. For $f \in C(\mathbb{R}), m \geq 2, l=1, \ldots, m-1$, it holds

$$
\left(Q_{h, m} f\right)^{(l)}(x)=\sum_{j \in \mathbb{Z}}\left(\sum_{k \in \mathbb{Z}} a_{j-k, m} h^{-l} \Delta_{h}^{l} f_{k}\right) B_{m-l}\left(h^{-1} x-j\right), \quad x \in \mathbb{R},
$$

where $\Delta_{h}^{l}=\left(\Delta_{h}\right)^{l}, \Delta_{h} f_{k}:=f_{k}-f_{k-1}=f\left(\left(k+\frac{m}{2}\right) h\right)-f\left(\left(k-1+\frac{m}{2}\right) h\right)$ is the backward difference of the function $f$, and $a_{k, m}$ are defined in (18).

Proof. With $d_{j}=\sum_{k \in \mathbb{Z}} a_{j-k, m} f_{k}, j \in \mathbb{Z}$, we get due to (6)

$$
\begin{aligned}
\left(Q_{h, m} f\right)^{\prime}(x) & =\frac{d}{d x} \sum_{j \in \mathbb{Z}}\left(\sum_{k \in \mathbb{Z}} a_{j-k, m} f_{k}\right) B_{m-1}\left(h^{-1} x-j\right) \\
& =\frac{d}{d x} \sum_{j \in \mathbb{Z}} d_{j} B_{m}\left(h^{-1} x-j\right) \\
& =h^{-1} \sum_{j \in \mathbb{Z}} d_{j}\left[B_{m-1}\left(h^{-1} x-j\right)-B_{m-1}\left(h^{-1} x-j-1\right)\right] \\
& =h^{-1} \sum_{j \in \mathbb{Z}}\left(d_{j}-d_{j-1}\right) B_{m-1}\left(h^{-1} x-j\right) \\
& =h^{-1} \sum_{j \in \mathbb{Z}}\left(\sum_{k \in \mathbb{Z}}\left(a_{j-k, m}-a_{j-1-k, m}\right) f_{k}\right) B_{m-1}\left(h^{-1} x-j\right) \\
& =h^{-1} \sum_{j \in \mathbb{Z}}\left(\sum_{k \in \mathbb{Z}} a_{j-k, m}\left(f_{k}-f_{k-1}\right)\right) B_{m-1}\left(h^{-1} x-j\right) \\
& =\sum_{j \in \mathbb{Z}}\left(\sum_{k \in \mathbb{Z}} a_{j-k, m} h^{-1} \Delta_{h} f_{k}\right) B_{m-1}\left(h^{-1} x-j\right) .
\end{aligned}
$$

Repeating the differentiations we obtain (35).

Lemma 5.2. For $m \geq 2, f \in V^{l, \infty}(\mathbb{R}), l=1, \ldots, m-1$, it holds

$$
\begin{aligned}
& \left\|\left(Q_{h, m} f\right)^{(l)}\right\|_{\infty} \leq \alpha_{m}\left\|f^{(l)}\right\|_{\infty} \\
& \left\|\left(Q_{h, m} f\right)^{(l)}\right\|_{\infty} \leq q_{m-l} \alpha_{m, l}\left\|f^{(l)}\right\|_{\infty},
\end{aligned}
$$

where $(c f .(15)) q_{m-l}=\left\|Q_{h, m-l}\right\|_{B C(\mathbb{R}) \rightarrow B C(\mathbb{R})}, b_{j, m-l}=B_{m-l}\left(j+\frac{m-l}{2}\right)$ and

$$
\alpha_{m}=\sum_{k \in \mathbb{Z}}\left|a_{k, m}\right|, \quad \alpha_{m, l}:=\sum_{k \in \mathbb{Z}}\left|\sum_{|j| \leq \operatorname{int}\{(m-l-1) / 2\}} a_{k-j, m} b_{j, m-l}\right|<\alpha_{m} .
$$


Proof. Since $\left|h^{-l} \Delta_{h}^{l} f_{k}\right| \leq\left\|f^{(l)}\right\|_{\infty}$, equality (35) implies (36):

$$
\begin{aligned}
\left\|\left(Q_{h, m} f\right)^{(l)}\right\|_{\infty} & \leq \sup _{j \in \mathbb{Z}}\left|\sum_{k \in \mathbb{Z}} a_{j-k, m} h^{-l} \Delta_{h}^{l} f_{k}\right| \\
& \leq \sup _{j \in \mathbb{Z}} \sum_{k \in \mathbb{Z}}\left|a_{j-k, m}\right| \sup _{k \in \mathbb{Z}}\left|h^{-l} \Delta_{h}^{l} f_{k}\right| \\
& =\sup _{j \in \mathbb{Z}} \sum_{k \in \mathbb{Z}}\left|a_{j-k, m}\right|\left\|f^{(l)}\right\|_{\infty} \\
& \leq \alpha_{m}\left\|f^{(l)}\right\|_{\infty} .
\end{aligned}
$$

According to (35), the spline $g:=\left(Q_{h, m} f\right)^{(l)} \in S_{h, m-l}$ has the knot values

$$
\begin{aligned}
g\left(\left(i+\frac{m-l}{2}\right) h\right) & =\sum_{j \in \mathbb{Z}}\left(\sum_{k \in \mathbb{Z}} a_{j-k, m} h^{-l} \Delta_{h}^{l} f_{k}\right) b_{i-j, m-l} \\
& =\sum_{k \in \mathbb{Z}}\left(\sum_{j \in \mathbb{Z}} a_{j-k, m} b_{i-j, m-l}\right) h^{-l} \Delta_{h}^{l} f_{k} \\
& =\sum_{k \in \mathbb{Z}}\left(\sum_{j \in \mathbb{Z}} a_{i-j-k, m} b_{j, m-l}\right) h^{-l} \Delta_{h}^{l} f_{k} ;
\end{aligned}
$$

we changed the summation variable $j \mapsto j^{\prime}=i-j$ and wrote instead of $j^{\prime}$ again $j$. Thus,

$$
\begin{aligned}
\left|g\left(\left(i+\frac{m-l}{2}\right) h\right)\right| & \leq \sum_{k \in \mathbb{Z}}\left|\sum_{j \in \mathbb{Z}} a_{i-j-k, m} b_{j, m-l}\right|\left\|f^{(l)}\right\|_{\infty} \\
& =\sum_{k \in \mathbb{Z}}\left|\sum_{j \in \mathbb{Z}} a_{k-j, m} b_{j, m-l}\right|\left\|f^{(l)}\right\|_{\infty} \\
& =\alpha_{m, l}\left\|f^{(l)}\right\|_{\infty} ;
\end{aligned}
$$

we changed the summation variable $k \mapsto k^{\prime}=i-k$, wrote instead of $k^{\prime}$ again $k$, and we took into account that $b_{j, m-l}=0$ for $|j|>\operatorname{int}\left(\frac{m-l-1}{2}\right)$. Since $Q_{h, m-l} g=g$ for $g=Q_{h, m-l} f$, we arrive at estimate (37):

$\left\|\left(Q_{h, m} f\right)^{(l)}\right\|_{\infty}=\|g\|_{\infty}=\left\|Q_{h, m-l} g\right\|_{\infty} \leq q_{m-l} \sup _{i \in \mathbb{Z}}\left|g\left(\left(i+\frac{m-l}{2}\right) h\right)\right| \leq q_{m-l} \alpha_{m, l}\left\|f^{(l)}\right\|_{\infty}$.

Finally, since $a_{k, m}$ are of alternating signs and $b_{j, m-l} \geq 0, \sum_{j} b_{j, m-l}=1$, we get the strict inequality occuring in (38): $\alpha_{m, l}=\sum_{k}\left|\sum_{j} a_{k-j, m} b_{j, m-l}\right|<$ $\sum_{k} \sum_{j}\left|a_{k-j, m}\right| b_{j, m-l}=\sum_{j} \sum_{k}\left|a_{k-j, m}\right| b_{j, m-l}=\sum_{j} \sum_{k}\left|a_{k, m}\right| b_{j, m-l}=\alpha_{m}$. 
Theorem 5.3. For $f \in V^{m, \infty}(\mathbb{R}), m \geq 2, l=1, \ldots, m-1$, it holds

$$
\begin{aligned}
& \left\|f^{(l)}-\left(Q_{h, m} f\right)^{(l)}\right\|_{\infty} \leq \Phi_{m-l+1} \pi^{-(m-l)} h^{m-l}\left(1+\alpha_{m}\right)\left\|f^{(m)}\right\|_{\infty} \\
& \left\|f^{(l)}-\left(Q_{h, m} f\right)^{(l)}\right\|_{\infty} \leq \Phi_{m-l+1} \pi^{-(m-l)} h^{m-l}\left(1+q_{m-l} \alpha_{m, l}\right)\left\|f^{(m)}\right\|_{\infty}
\end{aligned}
$$

with constants $\alpha_{m}$ and $\alpha_{m, l}$ defined in (38).

Proof. Introduce the operator $P_{h, m, l}:=\partial^{l} Q_{h, m} K_{l}$ where $\partial^{l}=\left(\frac{d}{d x}\right)^{l}$ whereas $K_{l}: C(\mathbb{R}) \rightarrow C(\mathbb{R})$ is the integral operator defined by

$$
\left(K_{l} u\right)(x)=\frac{1}{(l-1) !} \int_{0}^{x}(x-t)^{l-1} u(t) d t, \quad x \in \mathbb{R} .
$$

Note that $f-K_{l} \partial^{l} f \in \mathcal{P}_{l-1}$ (it is the Taylor polynomial of $f$ ), hence $Q_{h, m}(f-$ $\left.K_{l} \partial^{l} f\right)=f-K_{l} \partial^{l} f, \partial^{l} Q_{h, m}\left(f-K_{l} \partial^{l} f\right)=0$ and

$$
f^{(l)}-\left(Q_{h, m} f\right)^{(l)}=f^{(l)}-\partial^{l} Q_{h, m} K_{l} f^{(l)}=\left(I-P_{h, m, l}\right) f^{(l)} .
$$

Further, $g \in S_{h, m-l}$ implies $K_{l} g \in S_{h, m}$ and $P_{h, m, l} g=\partial^{l} Q_{h, m} K_{l} g=\partial^{l} K_{l} g=g$. We can continue $f^{(l)}-\left(Q_{h, m} f\right)^{(l)}=\left(I-P_{h, m, l}\right)\left(f^{(l)}-Q_{h, m-l} f^{(l)}\right)$. Estimating with the help of Theorem 3.1 we obtain

$$
\left\|f^{(l)}-\left(Q_{h, m} f\right)^{(l)}\right\|_{\infty} \leq\left(1+\left\|P_{h, m, l}\right\|_{B C(\mathbb{R}) \rightarrow B C(\mathbb{R})}\right) \Phi_{m-l+1} \pi^{-(m-l)} h^{m-l}\left\|f^{(m)}\right\|_{\infty} .
$$

For $g \in B C(\mathbb{R})$, it holds $K_{l} g \in V^{l, \infty}(\mathbb{R}),\left(K_{l} g\right)^{(l)}=g$, and Lemma 3 implies $\left\|P_{h, m, l}\right\|_{B C(\mathbb{R}) \rightarrow B C(\mathbb{R})} \leq \alpha_{m}$ and $\left\|P_{h, m, l}\right\|_{B C(\mathbb{R}) \rightarrow B C(\mathbb{R})} \leq \alpha_{m, l}$ that completes the proof of estimates (39) and (40).

Remark 5.4. Using Remark 3.3 we obtain that for $f \in V^{l, \infty}(\mathbb{R})$ with $f^{(l)} \in$ $B U C(\mathbb{R}), 0<l<m$, it holds $\left\|f^{(l)}-\left(Q_{h, m} f\right)^{(l)}\right\|_{\infty} \rightarrow 0$ as $h \rightarrow 0$.

\section{Interpolation of modestly smooth functions}

Now we discuss the error of the spline interpolant for less smooth functions compared with the main result (Theorem 3.1).

Theorem 6.1. For $m \geq 2, f \in V^{l, \infty}(\mathbb{R}), 1 \leq l \leq m-1$, it holds

$$
\begin{aligned}
& \left\|f-Q_{h, m} f\right\|_{\infty} \leq \Phi_{l+1} \pi^{-l} h^{l}\left(1+\alpha_{m}\right)\left\|f^{(l)}\right\|_{\infty} \\
& \left\|f-Q_{h, m} f\right\|_{\infty} \leq \Phi_{l+1} \pi^{-l} h^{l}\left(1+q_{m-l} \alpha_{m, l}\right)\left\|f^{(l)}\right\|_{\infty} ;
\end{aligned}
$$

for constants $\alpha_{m}$ and $\alpha_{m, l}$ see (38). If, in addition, $f^{(l)} \in B U C(\mathbb{R})$, then

$$
\left\|f-Q_{h, m} f\right\|_{\infty}=o\left(h^{l}\right) \quad \text { as } h \rightarrow 0 .
$$


Proof. Since $\left(Q_{h, m} f\right)\left(\left(i+\frac{m}{2}\right) h\right)=f\left(\left(i+\frac{m}{2}\right) h\right), i \in \mathbb{Z}$, we have

$$
\begin{aligned}
f-Q_{h, m} f & =\left(I-Q_{h, l}\right)\left(f-Q_{h, m} f\right) & & \text { for even } m-l \\
S_{h / 2}\left(f-Q_{h, m} f\right) & =\left(I-Q_{h, l}\right) S_{h / 2}\left(f-Q_{h, m} f\right) & & \text { for odd } m-l,
\end{aligned}
$$

where the shift operator $S_{h / 2}$ is defined by $\left(S_{h / 2} f\right)(x)=f\left(x-\frac{h}{2}\right)$. By Theorem 3.1, $\left\|f-Q_{h, m} f\right\|_{\infty} \leq \Phi_{l+1} \pi^{-l} h^{l}\left(\left\|f^{(l)}\right\|_{\infty}+\left\|\left(Q_{h, m} f\right)^{(l)}\right\|_{\infty}\right)$, and Lemma 5.2 completes the proof of estimates (41) and (42).

Assume that $f^{(l)} \in B U C(\mathbb{R})$ and estimate again with the help of Theorem 3.1: $\left\|f-Q_{h, m} f\right\|_{\infty} \leq \Phi_{l+1} \pi^{-l} h^{l}\left\|f^{(l)}-\left(Q_{h, m} f\right)^{(l)}\right\|_{\infty}=o\left(h^{l}\right)$ as asserted in (43). Here we took into account that by Remark $5.4\left\|f^{(l)}-\left(Q_{h, m} f\right)^{(l)}\right\|_{\infty} \rightarrow 0$ as $h \rightarrow 0$.

Theorem 6.2. Assume that $f \in C(\mathbb{R})$ is uniformly Hölder continuous on $\mathbb{R}$ :

$$
\left|f\left(x_{1}\right)-f\left(x_{2}\right)\right| \leq c_{f}\left|x_{1}-x_{2}\right|^{\theta} \quad \forall x_{1}, x_{2} \in \mathbb{R}
$$

where $0<\theta \leq 1$. Then

$$
\left\|f-Q_{h, m} f\right\|_{\infty} \leq\left(2^{-\theta}+\frac{1}{2} \alpha_{m}\right) c_{f} h^{\theta}
$$

Proof. We have

$$
\begin{aligned}
f-Q_{h, m} f & =\left(I-Q_{h, 1}\right)\left(f-Q_{h, m} f\right) & & \text { for odd } m \\
S_{h / 2}\left(f-Q_{h, m} f\right) & =\left(I-Q_{h, 1}\right) S_{h / 2}\left(f-Q_{h, m} f\right) & & \text { for even } m .
\end{aligned}
$$

For $x \in[i h,(i+1) h), i \in \mathbb{Z}$, it holds $\left(Q_{h, 1} f\right)(x)=f\left(\left(i+\frac{1}{2}\right) h\right)$ and due to (44), $\left|f(x)-\left(Q_{h, 1} f\right)(x)\right|=\left|f(x)-f\left(\left(i+\frac{1}{2}\right) h\right)\right| \leq c_{f}\left(\frac{h}{2}\right)^{\theta}$, thus $\left\|f-Q_{h, 1} f\right\|_{\infty} \leq c_{f}\left(\frac{h}{2}\right)^{\theta}$. Similarly, establishing for $g:=Q_{h, m} f$ and for $g:=S_{h / 2} Q_{h, m} f$ an estimate

$$
\left|g(x)-g\left(\left(i+\frac{1}{2}\right) h\right)\right| \leq c_{g}\left(\frac{h}{2}\right)^{\theta}, \quad x \in[i h,(i+1) h), i \in \mathbb{Z},
$$

we have $\left\|g-Q_{h, 1} g\right\|_{\infty} \leq c_{g}\left(\frac{h}{2}\right)^{\theta}$, and $\left\|f-Q_{h, m} f\right\|_{\infty} \leq\left(c_{f}+c_{g}\right)\left(\frac{h}{2}\right)^{\theta}$. So it remains to establish (46). For $x \in[i h,(i+1) h), i \in \mathbb{Z}$, it holds $\left|g(x)-g\left(\left(i+\frac{1}{2}\right) h\right)\right| \leq$ $\left\|g^{\prime}\right\|_{\infty} \frac{h}{2}$. By Lemma 5.1,

$$
g^{\prime}(x)=\sum_{j \in \mathbb{Z}}\left(\sum_{k \in \mathbb{Z}} a_{j-k, m} h^{-1} \Delta_{h} f_{k}\right) B_{m-1}\left(h^{-1} x-j\right), \quad x \in \mathbb{R},
$$

that implies $\left\|g^{\prime}\right\|_{\infty} \leq h^{-1} \alpha_{m} \sup _{k \in \mathbb{Z}}\left|\Delta_{h} f_{k}\right| \leq h^{-1} \alpha_{m} c_{f} h^{\theta}$. Now (46) with $c_{g}=$ $2^{\theta-1} \alpha_{m} c_{f}$ and (45) follow. 


\section{An extension of the main result}

Introduce the space $V_{h}^{m, \infty}(\mathbb{R}), m \geq 2$, of functions $g \in C^{m-2}(\mathbb{R})$ such that the derivatives $g^{(m-1)}, g^{(m)}$ exist on every interval $(i h,(i+1) h)$ and $\left.g^{(m-1)}\right|_{(i h,(i+1) h)} \in$ $C((i h,(i+1) h)),\left.g^{(m)}\right|_{(i h,(i+1) h)} \in L^{\infty}((i h,(i+1) h)), i \in \mathbb{Z}$,

$$
\sigma_{h, m}(g):=\sup _{i \in \mathbb{Z}} \sup _{i h<x<(i+1) h)}\left|g^{(m)}(x)\right|<\infty .
$$

Clearly, $V^{m, \infty}(\mathbb{R}) \subset V_{h}^{m, \infty}(\mathbb{R})$ and $\left\|f^{(m)}\right\|_{\infty}=\sigma_{h, m}(f)$ for $f \in V^{m, \infty}(\mathbb{R})$.

Lemma 7.1. For $m \geq 2$, it holds $V_{h}^{m, \infty}(\mathbb{R})=V^{m, \infty}(\mathbb{R})+S_{h, m}$, i.e., every $g \in V_{h}^{m, \infty}(\mathbb{R})$ has a representation

$$
g=f+g_{h}, \quad f \in V^{m, \infty}(\mathbb{R}), g_{h} \in S_{h, m} .
$$

In particular, (47) holds with

$$
f(x)=\frac{1}{(m-1) !} \int_{0}^{x}(x-t)^{m-1} G_{m}(t) d t, \quad x \in \mathbb{R},
$$

where $G_{m} \in L^{\infty}(\mathbb{R})$ is defined by $G_{m}(x)=g^{(m)}(x)$ for $x \in(i h,(i+1) h), i \in \mathbb{Z}$ (and other $f$ in (47) differ from (48) by an additive polynomial of degree $m-1$ ).

Proof. Let $g \in V_{h}^{m, \infty}(\mathbb{R})$ and let $f$ be defined by (48). Then $f^{(m)}=G_{m} \in$ $L^{\infty}(\mathbb{R})$, hence $f \in V^{m, \infty}(\mathbb{R})$ and $f^{\prime}, \ldots, f^{(m-1)}$ are continuous in $\mathbb{R}$. Together with $g$, also $g_{h}:=g-f \in C^{m-2}(\mathbb{R})$. Further, since $\left.g^{(m-1)}\right|_{(i h,(i+1) h)} \in C((i h,(i+$ 1)h) $),\left.g^{(m)}\right|_{(i h,(i+1) h)}=G_{m} \in L^{\infty}((i h,(i+1) h))$, we have

$$
\begin{aligned}
g_{h}^{(m-1)}(x) & =g^{(m-1)}(x)-f^{(m-1)}(x) \\
& \left.=g^{(m-1)}\left(i+\frac{1}{2}\right) h\right)+\int_{\left(i+\frac{1}{2}\right) h}^{x} G_{m}(t) d t-\int_{0}^{x} G_{m}(t) d t \\
& \left.=g^{(m-1)}\left(i+\frac{1}{2}\right) h\right)-\int_{0}^{\left(i+\frac{1}{2}\right) h} G_{m}(t) d t, \quad x \in(i h,(i+1) h), i \in \mathbb{Z} .
\end{aligned}
$$

Thus $g_{h}^{(m-1)}(x)$ is constant on the intervals $(i h,(i+1) h), i \in \mathbb{Z}$, i.e., $\left.g_{h}\right|_{(i h,(i+1) h)} \in$ $\mathcal{P}_{m-1}, i \in \mathbb{Z}$, and $g_{h} \in S_{h, m}$.

Theorem 7.2. Let $m \geq 2$. Assume that $g \in V_{h}^{m, \infty}(\mathbb{R})$ satisfies the inequality

$$
|g(x)| \leq c\left(1+|x|^{r}\right), \quad x \in \mathbb{R},
$$

where $r \geq 0$ and $c \geq 0$. Then

$$
\begin{aligned}
\left|g(x)-\left(Q_{h, m} g\right)(x)\right| & \leq \sigma_{h, m}(g)\left|E_{h, m+1}(x)\right|, \quad x \in \mathbb{R} \\
\left\|g-Q_{h, m} g\right\|_{\infty} & \leq \Phi_{m+1} \pi^{-m} h^{m} \sigma_{h, m}(g) .
\end{aligned}
$$


Proof. Let $f \in V^{m, \infty}(\mathbb{R})$ and $g_{h} \in S_{h, m}$ be defined by (47), (48). Then $|f(x)| \leq \frac{1}{m !}\left\|G_{m}\right\|_{\infty}|x|^{m}, x \in \mathbb{R}$, that together with (49) implies $\left|g_{h}(x)\right| \leq$ $c^{\prime}\left(1+|x|^{\max \{m, r\}}\right), x \in \mathbb{R}$. Hence $Q_{h, m} f, Q_{h, m} g$ and $Q_{h, m} g_{h}$ are well defined, and $Q_{h, m} g_{h}=g_{h}$. Equality (47) yields $\left(I-Q_{h, m}\right) g=\left(I-Q_{h, m}\right) f$. According to Theorem 3.1, $\left(I-Q_{h, m}\right) f$ is estimated by (26), (27) which take the form (50), (51) since $\left\|f^{(m)}\right\|_{\infty}=\left\|G_{m}\right\|_{\infty}=\sigma_{h, m}(g)$.

In the case of 1 -periodic $f$ and $h=\frac{1}{n}$ with an even $n \in \mathbb{N}$, Theorem 7.2 is equivalent to a result of [2].

Acknowledgement. This work was supported by Estonian Science Foundation (research grant No. 5859).

\section{References}

[1] de Boor, C., A Practical Guide to Splines. New York: Springer 2001.

[2] Korneychuk, N. P., Splines in the Approximation Theory (in Russian). Moscow: Nauka 1984.

[3] Leetma, E. and Vainikko, G., Quasi-interpolation by splines on the uniform knot sets. Math. Model. Anal. 12 (2007), $107-120$.

[4] Schoenberg, I. J., Cardinal interpolation and spline functions. J. Approxim. Theory 2 (1969), 167 - 206.

[5] Schoenberg, I. J., Cardinal interpolation and spline functions. II. Interpolation of data of power growth. J. Approxim. Theory 6 (1972), $404-420$.

[6] Schoenberg, I. J., Cardinal Spline Interpolation. Philadelphia (PA): SIAM 1973.

[7] Schumaker, L. L., Spline Functions: Basic Theory. Malabar (FL): Krieger 1993.

[8] Stechkin, S. B. and Subbotin, Yu. N., Splines in Numerical Mathematics (in Russian). Moscow: Nauka 1976.

[9] Vainikko, G., On the best approximation of function classes from values on a uniform grid in the real line. WSEAS Trans. Math. 6 (2007), 523 - 528.

[10] Zav'yalov, Yu. S., Kvasov, B. I. and Miroshnichenko, V. L., Methods of Spline Functions (in Russian). Moscow: Nauka 1980.

[11] Zygmund, A., Trigonometric Series 1. Cambridge: Cambridge Univ. Press 1959.

Received April 24, 2007 